\title{
Enfermedad pulmonar obstructiva crónica durante la pandemia de coronavirus 2020
}

\author{
Chronic obstructive pulmonary disease \\ during the 2020 coronavirus pandemic
}

\begin{abstract}
Germán díaz-Santos, MD¹, Manuel Pacheco, MD ${ }^{1}$, Milena García, MD¹, Rodrígo Rubio Rubio, MD ${ }^{1}$, Rubén Darío Contreras, MD¹, Wihen Ariza, MD¹, Rafael Acero, MD¹, Tulio LÁZaro, MD¹, José Ezequiel Mier

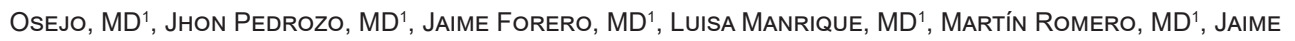
Alvarado, MD ${ }^{1}$, Carlos Aguirre, MD ${ }^{1}$, Robin Rada, MD ${ }^{1}$, IVÁn de Jesús Baños Álvarez, MD'1 Carlos Celis, MD ${ }^{1}$, Ana Catalina Vanegas, MD ${ }^{1}$, Carlos Matiz, MD ${ }^{1}$, Gustavo Adolfo Hincapié Díaz MD ${ }^{1}$, Alejandro Casas MD ${ }^{1}$, ABraham Alí MUNIVE, MD ${ }^{1}$
\end{abstract}

\section{Introducción}

Desde la aparición del brote en China, se ha informado que la neumonía por coronavirus impacta más la mortalidad en pacientes adulto mayores y con comorbilidades, entre la cual está la enfermedad pulmonar obstructiva crónica (EPOC) y el tabaquismo. En Colombia, el primer caso apareció el 6 de marzo de 2020, y el riesgo de contraer la enfermedad en este país es alto. Se reconoce que las personas con EPOC se encuentran entre las más afectadas por COVID-19, y debe tratarse de minimizar el impacto de la infección (1).

Aunque no fue tan alta la cantidad de pacientes con EPOC que se infectaron con coronavirus en Wuhan, la mayoría de estos requirió ingresar a unidad de cuidados intensivos (UCI) (2). Durante esta pandemia, la prevención de la nueva neumonía por COVID-19 en pacientes con EPOC se ha convertido en una prioridad para las diferentes asociaciones, concientizando al paciente con EPOC. Estos individuos tienen 6,4 veces más probabilidad de desarrollar una enfermedad grave, y 17,8 veces más probabilidades de necesitar manejo en la unidad de cuidado intensivo que la población general (3).

El objetivo de este documento es resumir la evidencia de estos últimos meses de infección y pandemia, y mostrar la opinión de los expertos, para ofrecer recomendaciones sobre la asociación del COVID-19 y la EPOC.

\section{Enfermedad pulmonar obstructiva crónica}

La EPOC es una entidad prevenible y tratable, que se caracteriza por una limitación al flujo aéreo persistente. Esta generalmente es progresiva y se asocia con una respuesta inflamatoria exagerada de las vías aéreas y del parénquima pulmonar frente a partículas o gases nocivos (4).

La prevalencia de EPOC en Colombia es de $8,9 \%$, con diferencias entre las ciudades como Medellín (13,5\%), Bogotá $(8,5 \%)$, Cali (8,5\%), Bucaramanga (7,9\%) y Barranquilla (6,2\%) (5).

\author{
${ }^{1}$ Medico Especialista en Medicina Inter- \\ na y Neumología, Miembro del Comité \\ de Enfermedad Pulmonar Obstructiva \\ Crónica de la Asociación Colombiana \\ de Neumología \\ Autor de correspondencia: \\ Germán Díaz-Santos. \\ Correo electrónico: \\ docger25@hotmail.com \\ Recibido: $28 / 04 / 2020$ \\ Aceptado: 30/05/2020
}




\section{Neumonía por coronavirus}

La neumonía grave, causada por la familia del coronavirus, a menudo se asocia con una rápida replicación y por una alta respuesta celular inflamatoria por citocinas y quimiocinas proinflamatorias elevadas, que terminan en un daño pulmonar agudo (DPA) y en síndrome de dificultad respiratoria aguda (SDRA) (6).

\section{EPOC frente a neumonía por coronavirus}

Tanto la EPOC estable, como la infección por coronavirus presentan similitudes y diferencias que nos pueden orientar. Algunas de estas se muestran en la Tabla 1.
La EPOC es una enfermedad crónica y requiere un tratamiento a largo plazo, que debe mantenerse durante todo el tiempo de la pandemia; a estas enfermedades crónicas se les puede prescribir por 3 meses o más. Las pruebas de rutina de la función pulmonar y de imagen pueden posponerse hasta que el Ministerio de Salud dé el aval y la seguridad de que la pandemia este controlada (7).

Se recomienda la inhalación regular de broncodilatadores de acción prolongada y la terapia con glucocorticoides/broncodilatadores para pacientes con EPOC estable, para la prevención de exacerbaciones agudas de EPOC (7).

Tabla 1. Comparación entre EPOC y COVID-19

\begin{tabular}{|c|c|c|}
\hline & EPOC & COVID-19 \\
\hline Presentación & $\begin{array}{l}\text { Empeoramiento agudo de los síntomas } \\
\text { respiratorios }\end{array}$ & Enfermedad respiratoria aguda \\
\hline Antecedentes & $\begin{array}{l}\text { - Tabaquismo } \\
\text { - Exposición crónica a humo } \\
\text { - Riesgos ocupacionales } \\
\text { - Enfermedad respiratoria previa o actual }\end{array}$ & $\begin{array}{l}\text { - Historia de viajes o residencia en países, } \\
\text { áreas o territorios que reporten transmisión } \\
\text { local de enfermedad por COVID-19 } \\
\text { - Contacto con paciente infectado } \\
\text { - Ninguna otra etiología que explique total- } \\
\text { mente la presentación clínica }\end{array}$ \\
\hline Clínica & $\begin{array}{l}\text { Disnea (progresiva, persistente y que empeora } \\
\text { con el esfuerzo); tos crónica, puede ser } \\
\text { intermitente o diaria; aumento del volumen y } \\
\text { cambio en la coloración (purulencia) } \\
\text { - Anorexia y pérdida de peso (estadios } \\
\text { avanzados de la enfermedad) }\end{array}$ & $\begin{array}{l}\text { Tos, malestar general, dolor de garganta, } \\
\text { fiebre o escalofrío, congestión nasal, dolor de } \\
\text { cabeza, dificultad respiratoria, dolor muscular, } \\
\text { diarrea, dolor de articulaciones, dolor de pecho, } \\
\text { náuseas/vómitos, irritabilidad o confusión, dolor } \\
\text { abdominal }\end{array}$ \\
\hline $\begin{array}{l}\text { Diagnóstico } \\
\text { No se deben } \\
\text { realizar estudios de } \\
\text { función pulmonar } \\
\text { en época de } \\
\text { pandemia por } \\
\text { COVID-19 }\end{array}$ & $\begin{array}{l}\text { - Clínica (antecedentes, signos, síntomas) } \\
\text { - Pruebas de función pulmonar } \\
\text { - Otras pruebas complementarias: detección de } \\
\alpha_{1} \text {-antitripsina, hemograma, radiografía de tórax } \\
\text { (PA y lateral) } \\
\text { - Descartar otras enfermedades asociadas }\end{array}$ & $\begin{array}{l}\text { - Hisopado nasofaríngeo u orofaríngeo } \\
\text { - Aspirado traqueal } \\
\text { - Lavado broncoalveolar no broncoscópico o } \\
\text { miniBAL } \\
\text { - Inicialmente no se requiere radiografía }\end{array}$ \\
\hline $\begin{array}{l}\text { Tratamiento } \\
\text { No se recomiendan } \\
\text { las nebulizaciones } \\
\text { durante la } \\
\text { pandemia, } \\
\text { por riesgo de } \\
\text { aerolización }\end{array}$ & $\begin{array}{l}\text { - Anticolinérgicos o } \beta_{2} \text {-agonistas de corta acción } \\
\text { Si se están utilizando broncodilatadores de } \\
\text { larga acción (LAMA, LABA o combinaciones), } \\
\text { continuarlos } \\
\text { - Inicie o aumente la frecuencia de la medica- } \\
\text { ción de rescate (salbutamol o ipratropio). Si su } \\
\text { médico le ha dado indicaciones adicionales de } \\
\text { medicamentos en caso de exacerbación, inície- } \\
\text { las sin retraso y comuníquese con él o con su } \\
\text { programa de atención, de ser posible }\end{array}$ & $\begin{array}{l}\text { - No existe un tratamiento específico para } \\
\text { el coronavirus. Aún no se ha demostrado } \\
\text { utilidad de antirretrovirales, ni existe vacuna } \\
\text { disponible en Colombia } \\
\text { - No existe suficiente información para sugerir } \\
\text { el inicio o suspensión de los medicamentos } \\
\text { tipo enalapril o losartán } \\
\text { - No hay suficiente evidencia para recomendar } \\
\text { el uso rutinario de la combinación de hidroxi- } \\
\text { cloroquina y azitromicina, ni ivermectina }\end{array}$ \\
\hline
\end{tabular}

PA: posteroanterior. 


\section{Exacerbación aguda de la EPOC y EPOC con neumonía por coronavirus}

En términos generales, la exacerbación aguda de los pacientes con EPOC se manifiesta como un aumento de los síntomas respiratorios, como la tos, el esputo y la disnea en un corto tiempo. Pero, en la etapa inicial de la neumonía por coronavirus predominan los síntomas sistémicos, como la fiebre y la disnea, con progresión rápida entre 6 a 7 días. La EPOC también es susceptible a neumonía adquirida en la comunidad (NAC), y siempre se debe plantear como un diagnóstico diferencial (7).

Los pacientes con EPOC infectados con COVID-19 no son fáciles de detectar, por lo cual, posiblemente tengan mayor mortalidad, es por esto por lo que nuestra mejor arma es la prevención. No existe evidencia científica que respalde que los corticoides inhalados (u orales) no puedan darse en pacientes con EPOC durante la pandemia de COVID-19 (1). De igual forma, se recomienda revisar la guía de Asoneumocito sobre exacerbación aguda de EPOC en la época de COVID-19.

\section{Prevención de la exacerbación aguda de la EPOC durante la pandemia}

Es muy importante prevenir la exacerbación aguda de la EPOC durante la pandemia. En la Tabla 2 se presentan algunas recomendaciones para esto.

Establezca un plan de manejo con el paciente y los familiares, si es posible, en caso de exacerbación de la EPOC (aumento de la tos, la expectoración, cambio de color de las flemas), sin que necesariamente se relacione con COVID-19. Explique que si se presenta fiebre persistente (con termómetro), aparición o aumento significativo de la sensación de falta de aire o hinchazón en las piernas, desorientación, confusión o color azul en labios o dedos, pida una cita prioritaria o consulte a Urgencias. Brinde información sobre las líneas telefónicas de atención: 123 desde teléfono fijo, 192 desde el celular. Pregunte por las líneas de atención de la EPS del paciente.
Tabla 2. Principales recomendaciones para la prevención de la exacerbación aguda de la EPOC durante la pandemia de COVID-19

Insista a su paciente que no suspenda los medicamentos, ni el oxígeno. No se debe abandonar el domicilio, si no es absolutamente necesario (utilizar tapabocas y evitar las aglomeraciones), y que se mantenga activo (ofrezca recomendaciones $u$ opciones de ejercicios en casa). Recomiende mantener la hidratación, y que consuma alimentos sanos, lo que permite controlar el peso

El uso de ventilación no invasiva (BiPAP, ASV) o CPAP favorece la transmisión del virus, si el paciente ya lo tiene; por lo cual, es recomendable que se mantenga en cuarentena estricta durante 2 semanas, esto para proteger a los habitantes de la casa

Eduque e insista en el lavado de manos vigorosamente con agua y jabón cada 3 horas. Puede usarse alcohol gel para remplazar el lavado de manos, si por alguna razón no tiene acceso a agua y jabón

Se recomienda (si no lo ha hecho) abandonar el cigarrillo, vacunación contra la gripe (disponible solo algunos meses del año) y la vacuna antineumocócica; mejorar la adherencia y técnicas de inhalación tanto de broncodilatadores de corta acción, como de broncodilatadores de acción prolongada o combinaciones

\section{Referencias}

1. Global Initiative for Chronic Obstructive Lung Disease [Internet]. GOLD COVID-19 guidance. Última visita: 25 de marzo de 2020. Disponible en: https://goldcopd.org/gold-covid19-guidance/

2. Wang D, Hu B, Hu C, Zhu F, Liu X, Zhang J, et al. Clinical characteristics of 138 hospitalized patients with 2019 novel coronavirus-infected pneumonia in Wuhan, China. JAMA. 2020;323(11):1061-9. doi:10.1001/jama.2020.1585.

3. Jain V, Yuan JM. Systematic review and meta-analysis of predictive symptoms and comorbidities for severe COVID-19 infection. Int J Public Health. 2020;65:533-46. doi: 10.1007/ s00038-020-01390-7.

4. Global Initiative for Chronic Obstructive Lung Disease. 2020 global strategy for prevention, diagnosis and management of COPD. Última visita: 25 de marzo de 2020. Disponible en: https://goldcopd.org/wp-content/uploads/2019/12/GOLD2020-FINAL-ver1.2-03Dec19_WMV.pdf 
5. Caballero A, Torres-Duque CA, Jaramillo C, Bolívar F, Sanabria F, Osorioet $\mathrm{P}$, et al. Prevalence of COPD in five Colombian cities situated at low, medium and high altitude (PREPOCOL study). Chest. 2008;133;343-9. doi: 10.1378/ chest.07-1361.

6. Channappanavar R, Perlman S. Pathogenic human coronavirus infections: causes and consequences of cytokine storm and immunopathology. Semin Immunopathol. 2017;39(5):52939. doi: 10.1007/s00281-017-0629-x.
7. Chronic Obstructive Pulmonary Disease Group of Chinese Thoracic Society; Chronic Obstructive Pulmonary Disease Committee of Chinese Association of Chest Physician. [Medical management and prevention instruction of chronic obstructive pulmonary disease during the coronavirus disease 2019 epidemic]. Zhonghua Jie He He Hu Xi Za Zhi. 2020;43(5):4216. doi: 10.3760/cma.j.cn112147-20200227-00201. 